\title{
Risk Factors for Systemic Inflammatory Response Syndrome in Patients with Negative Preoperative Urine Culture after Percutaneous Nephrolithotomy
}

\author{
Ekrem Akdeniz ${ }^{1}$, Kemal Ozturk ${ }^{1}$, Muhammet Bahaettin Ulu ${ }^{1}$, Metin Gur $^{1}$, Suleyman Tumer Caliskan ${ }^{1}$ and Emine \\ Sehmen ${ }^{2}$ \\ ${ }^{1}$ Department of Urology, Gazi Hospital, Samsun, Turkey \\ ${ }^{2}$ Department of Infectious Diseases, Gazi Hospital, Samsun, Turkey
}

\begin{abstract}
Objective: To explore the risk factors for systemic inflammatory response syndrome (SIRS) after percutaneous nephrolithotomy (PCNL) in patients with preoperative negative urine culture (UC).

Study Design: Observational study.

Place and Duration of Study: Department of Urology, Gazi Hospital, Samsun, Turkey, from January 2015 to January 2020.

Methodology: Two hundred and twenty-eight patients, who underwent conventional PCNL for renal stones, were evaluated. The patients were divided into non-SIRS (Group 1) and SIRS (Group 2) groups, and the effects of the variables were investigated to predict the development of SIRS.

Results: Despite preoperative sterile UC, SIRS developed postoperatively in 29 (12.7\%) patients. The univariate analysis revealed a statistically significant difference between groups in preoperative serum C-reactive protein (CRP) $(p<0.001)$, platelet-to-lymphocyte ratio (PLR) $(p<0.001)$, neutrophil-to-lymphocyte ratio $(p=0.001)$, urine white blood cell $(p=0.034)$, stone size $(p=0.023)$, operative time $(p=0.041)$, hemoglobin drop $(p<0.001)$, blood transfusion $(p=0.002)$, hospital stay ( $p$ $=0.006)$, and complication rate $(p=0.001)$. Receiver operating characteristic analysis indicated that PLR $>117.36(p<0.001)$, CRP $>3.16 \mathrm{mg} / \mathrm{L}(p<0.001)$, stone burden $>471 \mathrm{~mm}^{2}(p=0.023)$ and hemoglobin drop $>2.3 \mathrm{~g} / \mathrm{L}(p<0.001)$ are independent risk factors for post-operative SIRS after PCNL.

Conclusion: PLR, CRP, stone size, and hemoglobin drop can predict SIRS after PCNL. This finding may help classify risk in patients before PCNL, especially in those with a sterile urine culture.

Key Words: C-reactive protein, Percutaneous nephrolithotomy, Platelet/lymphocyte ratio, Systemic inflammatory response syndrome.

How to cite this article: Akdeniz E, Ozturk K, Ulu MB, Gur M, Caliskan ST, Sehmen E. Risk Factors for Systemic Inflammatory Response Syndrome in Patients with Negative Preoperative Urine Culture after Percutaneous Nephrolithotomy. J Coll Physicians Surg Pak 2021; 31(04):410-416.
\end{abstract}

\section{INTRODUCTION}

Percutaneous nephrolithotomy (PCNL) is the gold standard method in the treatment of complex, large or multiple kidney stones. ${ }^{1}$ As with all surgical procedures, various complications may occur in PCNL. Despite developments in technology and advancement of surgical techniques, the most common cause of mortality in PCNL is sepsis, which develops due to postoperative infection. ${ }^{2}$ Sepsis occurs in $0.3 \%$ to $1.1 \%$ of patients, and reported mortality rate ranges from $50 \%$ to $66 \%$. $^{3.5}$

Correspondence to: Dr. Ekrem Akdeniz, Department of Urology, Gazi Hospital, Samsun, Turkey

E-mail: ekrem.akdeniz@saglik.gov.tr

Received: January 30, 2021; Revised: February 23, 2021;

Accepted: March 05, 2021

DOI: https://doi.org/10.29271/jcpsp.2021.04.410
A number of factors have, therefore, been identified in predicting the development of systemic inflammatory response syndrome (SIRS) after PCNL, including positive urine culture (UC), gender, hydronephrosis, stone complexity, multiple access, surgical time, residual stones, blood transfusion, staghorn stone, and stone burden. ${ }^{6}$ However, many urologists rely on preoperative midstream UC to assess the risk of SIRS after PCNL due to some discrepancies in these risk factors identified in studies conducted. ${ }^{7}$ However, preoperative midstream UC is not very effective in predicting SIRS because preoperative UC was found to be negative in $36.8-52.4 \%$ of patients, who developed sepsis after PCNL. ${ }^{8,9}$ In this case, new markers are needed to evaluate the risk of postoperative SIRS.

SIRS is a syndrome, causing both infectious and non-infectious inflammation, and it is closely associated with sepsis. ${ }^{10}$ In inflammatory diseases, peripheral blood cell numbers vary and thus blood cell-based biomarkers, such as platelet-to-lymphocyte ratio (PLR) and neutrophil-to-lymphocyte ratio (NLR) are 
formed. These biomarkers are used in predicting the diagnosis and treatment of a large number of diseases. ${ }^{11}$ In previously conducted studies, it has been found that inflammation markers such asPLR, NLR and acute phase reactant suchasC-reactive protein (CRP) are independent risk factors in predicting SIRS development after PCNL. ${ }^{11-14}$ However, studies conducted on the development of postoperative SIRS in patients with preoperative sterile UC are very limited. In this study, patients with negative preoperative midstream UC, who developed SIRS in postoperative period, were evaluated and the aim was to show different markers in predicting SIRS development after PCNL.

\section{METHODOLOGY}

After the Ethics Committee approval was obtained, the data of patients between January 2015 and January 2020, and who developed SIRS in postoperative period, were evaluated retrospectively in the clinic at Department of Urology, Gazi Hospital, Samsun, Turkey. Anamnesis, physical examination, and demographic features were recorded for patients whose informed surgical consent forms were taken prior to surgery. Non-contrast computed tomography (CT) examination was performed on all patients as a standard. The maximum length and width of each stone was multiplied for the calculation of stone size, which was expressed in $\mathrm{mm}^{2}$ Preoperative laboratories collected CRP, hemogram parameters, urine analysis, and urine culture results. CRP levels were measured by using nephelometric immunoassay, a part of the standard preoperative order set. The reference value of CRP was $0.00-5.00 \mathrm{mg} / \mathrm{L}$ according to the method studied at the study place. Clavien-Dindo classification was used to assess the complications. A close monitorisation of the vital signs was performed and postoperative blood counts were performed.

Based on the presence of postoperative SIRS, the patients were divided into a non-SIRS group (Group 1) and SIRS group (Group 2). International Sepsis Definition Conference-2001 criteria were used to determine the presence of SIRS. ${ }^{15}$ In case of meeting at least two or more of the following criteria, SIRS diagnosis was made: heart rate of $>90 \mathrm{bpm}$, respiratory rate of $>20$ breaths/min or $\mathrm{PaCO}_{2}$ of $<32 \mathrm{mmHg}$, body temperature of $<36^{\circ} \mathrm{C}$ or $>38^{\circ} \mathrm{C}$, and white blood cell (WBC) count of $>12.000 / \mathrm{mm}^{3}$ or $<4.000 / \mathrm{mm}^{3}$.

Patients were excluded, if they were younger than 18 years, had preoperative positive UC, had used preoperative antibiotics, had underwent bilateral PCNL in the same session, or had prePCNL drainage catheter (urethral catheter, ureteral stent or percutaneous nephrostomy). Patients with sheet channel size of $<24 \mathrm{~F}$ during PCNL, had underwent tubeles PCNL, or had underwent renal surgery previously, those with a tumour, those with chronic renal insufficiency, those who were using chronic oral immunosuppressive agents or those who had renal congenital malformations, were also excluded.

Demographic and clinical data, preoperative urine WBC, preoperative hemogram parameters, CRP, NLR, and PLR; decrease in hemoglobin, bloodtransfusion rate, stone-freestatus, complication rate, and hospital stay were analysed in both groups.

After receiving a single dose prophylactic antibiotic regimen intravenously, the patients underwent PCNL under general anesthesia. The collecting system was identified with a contrast agent; and an 18-gauge access needle was used under C-arm fluoroscopic guidance to create percutaneous access into the selected calyx. Following the insertion of a guide wire, serial fascial dilators between 26-30 Fr with an amplatz dilators were used to dilate the tract and access sheath was placed. Stone fragmentation was performed with 24 or 26 Fr nephroscope and pneumatic lithotriptor and extracted with stone forceps (Figure $1)$. Nephrostomy catheter was routinely placed when both procedures ended. On postoperative day 1 to 3 , the nephrostomy catheter was removed. Kidney, ureter, and bladderX-rays and biochemical tests were performed on postoperative day 1 to evaluate the patients. CT was used to evaluate final stonefree (SF) rate on postoperative day 15.

The data were analysed with 25 (Statistical Package for Social Sciences- IBM Corp., Armonk, NY, USA) programme. The results were considered as statistically significant for $p<0.05$. Nominal data were expressed as frequencies and percentages, while normally distributed continuous data were expressed as mean \pm standard deviation, and non-normally distributed continuous data as median (interquartile range [IQR]: $25^{\text {th }}$ percentile- $75^{\text {th }}$ percentile). Normality distribution of continuous variables was analysed with Kolmogorov-Smirnov test and Shapiro-Wilks test. Independent Samples t-test was used to evaluate whether there was statistically significant difference between the two groups in terms of normally distributed continuous variables, while Mann-Whitney U-test was used to evaluate whether there was statistically significant difference between the two groups in terms of continuous variables, which were not normally distributed. Nominal variables were evaluated with Chi-square test and Fisher's Exact test. Independent risk factors effective on postoperative SIRS development were found with binary logistic regression analysis. Odds ratio, 95\% confidence interval and significance levels of each risk factor were calculated. The threshold value with the highest sensitivity and specificity of variables was found for disease with receiver operating characteristic (ROC) analysis.

\section{RESULTS}

A total of 228 patients ( 76 females, 152 males) with median age of 48.5 (IQR: 36-58.75) years were included in the study. Twenty-five (11\%) patients were found to have diabetes mellitus (DM) and body mass index (BMI) of the patients was $28.77 \pm 4.21$ $\mathrm{kg} / \mathrm{m}^{2}$. While average stone area was 377 (IQR: 308.2-667.5) $\mathrm{mm}^{2}$ and average surgical time was 60 (IQR: 55-75) minutes. Average hospitalisation time of the patients was 3 (IQR: 2-3) days and SF rate was $81.6 \%$. CRP, NLR and PLR values of the patients were found as 1.65 (IQR: 0.91-2.96) mg/L, 2.34 (IQR: 1.86-2.76), and 111.16 (IQR: $90.1-135)$ respectively. The detailed characteristics in each group are listed in Tablel. 
Table I: Detailed information of all patients in the study.

\begin{tabular}{|c|c|c|c|c|}
\hline Variables & Overall $(\mathrm{N}=228)$ & Group I (N = 199) & Group II (N = 29) & p-value \\
\hline Age (years), median (IQR) & $48.5(36-58.75)$ & $48(35-58)$ & $53(43-63.5)$ & 0.078 \\
\hline $\begin{array}{l}\text { Gender, N (\%) } \\
\text { Female } \\
\text { Male }\end{array}$ & $\begin{array}{r}76(33.3 \%) \\
152(66.7 \%)\end{array}$ & $\begin{array}{c}65(32.7 \%) \\
134(67.3 \%)\end{array}$ & $\begin{array}{l}11(37.9 \%) \\
18(62.1 \%)\end{array}$ & 0.574 \\
\hline $\mathrm{BMI}\left(\mathrm{kg} / \mathrm{m}^{2}\right)$, mean $\pm \mathrm{SD}$ & $28.77 \pm 4.21$ & $28.86 \pm 4.3$ & $28.17 \pm 3.51$ & 0.408 \\
\hline History of diabetes, N (\%) & $25(11 \%)$ & $17(8.5 \%)$ & $8(27.6 \%)$ & 0.006 \\
\hline $\begin{array}{l}\text { Preoperative laboratories } \\
\text { Serum CRP, median (IQR), mg/L } \\
\text { PLR, median (IQR) } \\
\text { NLR, median (IQR) } \\
\text { Urine WBC, median (IQR), (cells per HPF) }\end{array}$ & $\begin{aligned} & 1.65(0.91-2.96) \\
& 111.16(90.1-135) \\
& 2.34(1.86-2.76) \\
& 7.5(3-16.75)\end{aligned}$ & $\begin{array}{c}1.4(0.88-2.45) \\
106.1(88.65-131.2) \\
2.25(1.83-2.68) \\
7(3-16)\end{array}$ & $\begin{array}{c}5.78(2.14-9.16) \\
135(121.3-164.88) \\
2.75(2.22-3.80) \\
13(6.5-24)\end{array}$ & $\begin{array}{c}<0.001 \\
<0.001 \\
0.001 \\
0.034\end{array}$ \\
\hline $\begin{array}{l}\text { Side of PNL, N (\%) } \\
\text { Right } \\
\text { Left }\end{array}$ & $\begin{array}{l}107(46.9 \%) \\
121(53.1 \%)\end{array}$ & $\begin{array}{c}97(48.7 \%) \\
102(51.3 \%)\end{array}$ & $\begin{array}{l}10(34.5 \%) \\
19(65.5 \%)\end{array}$ & 0.151 \\
\hline $\begin{array}{l}\text { Stone Location, N (\%) } \\
\text { Pelvis } \\
\text { Isolated calyx } \\
\text { Pelvis + Calyx } \\
\text { Staghorn }\end{array}$ & $\begin{array}{l}66(28.9 \%) \\
42(18.4 \%) \\
68(29.8 \%) \\
52(22.8 \%)\end{array}$ & $\begin{array}{c}60(30.2 \%) \\
34(17 \%) \\
62(31.2 \%) \\
43(21.6 \%)\end{array}$ & $\begin{array}{c}6(20.7 \%) \\
8(27.6 \%) \\
6(20.7 \%) \\
9(31 \%)\end{array}$ & 0.239 \\
\hline Stone size $\left(\mathrm{mm}^{2}\right)$, median (IQR) & $377(308.2-667.5)$ & $363(297-614)$ & $498(336-914)$ & 0.023 \\
\hline ASA score, median (IQR) & $2(1-2)$ & $2(1-2)$ & $2(1-2.5)$ & 0.008 \\
\hline $\begin{array}{l}\text { Access Number, N (\%) } \\
\text { Single } \\
\text { Multiple }\end{array}$ & $\begin{array}{r}184(80.7 \%) \\
44(19.3 \%)\end{array}$ & $\begin{array}{c}165(82.9 \%) \\
34(17.1 \%)\end{array}$ & $\begin{array}{l}19(65.5 \%) \\
10(34.5 \%)\end{array}$ & 0.027 \\
\hline Operative time (minute), median (IQR) & $60(55-75)$ & $60(55-70)$ & $65(60-96)$ & 0.041 \\
\hline Blood transfusion, $\mathrm{N}(\%)$ & $13(5.7 \%)$ & $7(3.5 \%)$ & $6(20.7 \%)$ & 0.002 \\
\hline Hemoglobin drop (g/dL), median (IQR) & $1.65(1.11-2.3)$ & $1.5(1.1-2.2)$ & $2.4(1.5-3.25)$ & $<0.001$ \\
\hline $\begin{array}{l}\text { Complication, N (\%) } \\
\text { Minor } \\
\text { Major }\end{array}$ & $\begin{array}{c}29(12.7 \%) \\
25(11 \%) \\
4(1.7 \%)\end{array}$ & $\begin{array}{c}19(9.5 \%) \\
17(8.5 \%) \\
2(1 \%)\end{array}$ & $\begin{array}{c}10(34.5 \%) \\
8(27.6 \%) \\
2(6.9 \%)\end{array}$ & 0.001 \\
\hline Hospital stay (day), median (IQR) & $3(2-3)$ & $3(2-3)$ & $3(2.5-4)$ & 0.006 \\
\hline Stone Free, $\mathrm{N}(\%)$ & $186(81.6 \%)$ & $165(82.9 \%)$ & $21(72.4 \%)$ & 0.173 \\
\hline
\end{tabular}

Table II. Multivariate analysis for predicting between patients with and without postoperative systemic inflammatory response syndrome.

\begin{tabular}{|c|c|c|c|c|}
\hline & p-value & Odds ratio (OR) & B & OR (95\% CI) Lower-Upper \\
\hline PLR ratio & 0.016 & 1.015 & 0.015 & $1.003-1.028$ \\
\hline CRP & 0.007 & 1.165 & 0.153 & $1.043-1.302$ \\
\hline Diabetes mellitus & 0.048 & 3.914 & 1.364 & $1.014-15.103$ \\
\hline Stone size & 0.040 & 1.001 & 0.001 & $1.000-1.003$ \\
\hline Hemoglobin drop & 0.022 & 1.742 & 0.555 & $1.082-2.803$ \\
\hline
\end{tabular}

Despite negative UC and broad exclusion criteria, SIRS developed in 29 (12.7\%) patients during post-operative hospitalization time. Causes of SIRS in patients meeting the SIRS criteria were WBC changes in $10(34.5 \%)$ patients, WBC + heart rate changes in four $(13.8 \%)$, heart rate + temperature + respiratory rate changes in four (13.8\%), $\mathrm{WBC}+$ heart rate + temperature changes in four $(13.8 \%)$, heart rate + temperature changes in three $(10.3 \%)$, WBC + heart rate + respiratory rate changes in two $(6.9 \%)$ and heart rate changes in two (6.9\%). According to ClavienDindo classification system, Grade 2 and higher complication developed in 29 patients. These were blood transfusion (Grade 2) in 13 patients, antibiotics (Grade 2) in 9 patients, antihypertensive therapy (Grade 2), and double J stent placement due to prolonged urine leakage (Grade 3a) in 2 patients each; nasogastric catheter application due to ileus (Grade 2); endoscopic ureter stone surgery (Grade 3b); and sepsis (Grade 4b) in one patient each.
When the patients were grouped in two as those who developed SIRS and those who did not develop SIRS and compared, no statistically significant difference was found between the groups in terms of age, gender, BMI, stone direction, stone localization, and SF rate. Significant difference was found between the groups when DM $(p=0.006)$, $\operatorname{CRP}(p<0.001), \operatorname{PLR}(p<0.001), \operatorname{NLR}(p=0.001)$, urine WBC count $(p=0.034)$, stone size $(p=0.023)$, mean American Society of Anesthesiologists (ASA) score $(p=0.008)$, access number $(p=0.027)$, operative time $(p=0.041)$, mean hemoglobin drop $(p<0.001)$, blood transfusion $(p=0$. $002)$, complication rate $(p=0.001)$, and hospital stay $(p=$ 0.006 ) values were compared.

Multivariate regression analysis revealed that PLR (OR = 1.015, 95\% Cl: 1.003-1.028, $p=0.016), C R P(O R=1.165$, $95 \% \mathrm{Cl}: 1.043-1.302, \mathrm{p}=0.007), \mathrm{DM}(\mathrm{OR}=3.914,95 \% \mathrm{Cl}$ : $1.014-15.103, p=0.048)$, stone size $(O R=1.001,95 \% \mathrm{Cl}$ : 
$1.000-1.003, p=0.04)$, and hemoglobin drop (OR $=1.742$, $95 \% \mathrm{Cl} 1.082-2.803, \mathrm{p}=0.022$ ) were independent factors affecting the post-operative development of SIRS after PCNL with a sterile baseline urine culture (Table II).

The ROC analysis of PLR to discriminate between groups showed an AUC of 0.753 (95\% Cl: 0.664-0.842). The optimal threshold value for PLR was 117.36 which had a sensitivity, specificity, positive predictive value (PPV), and negative predictive value (NPV) of $89.7 \%, 63.8 \%, 26.5 \%$, and $97.7 \%$, respectively. ROC analysis of CRP value to discriminate between groups showed an AUC of $0.802(95 \% \mathrm{Cl}$ : 0.716-0.888). The CRP cut-off value from the ROC analysis was $3.16 \mathrm{mg} / \mathrm{L}$ which had a sensitivity, specificity, PPV, and NPV of $65.5 \%, 84.9 \%, 38.8 \%$, and $94.4 \%$ respectively.

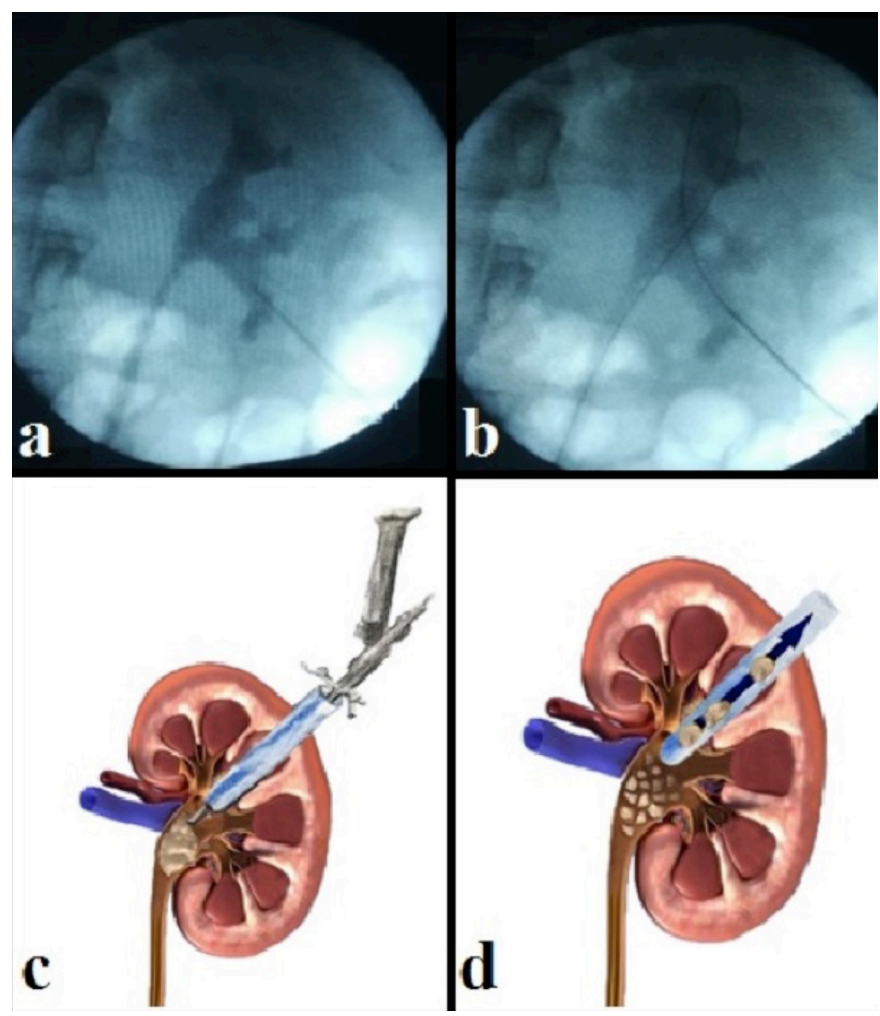

Figure 1: The different stages of percutaneous nephrolithotomy, (a) Access to the renal collecting system is first established with a needle by means of a contrast agent, (b) Insertion of the guide wire into the collecting system, (c) A nephroscope is employed to locate the stone, which is subsequently fragmented using a lithotripter, (d) Finally, a grasper and the nephroscope are employed to remove any stone fragments.

ROC analysis of stone size to discriminate between groups showed an AUC of 0.631 (95\% Cl: $0.523-0.738)$. The stone size cut-off value from the ROC analysis was $471 \mathrm{~mm}^{2}$ which had a sensitivity, specificity, PPV, and NPV of $55.2 \%, 69.3 \%$, $20.8 \%$, and $91.4 \%$ respectively. ROC analysis of hemoglobin drop to discriminate between groups showed an AUC of 0.716 (95\% Cl: 0.615-0.817). The hemoglobin drop cut-off value from the ROC analysis was $2.3 \mathrm{~g} / \mathrm{dL}$ which had a sensitivity, specificity, PPV, and NPV of $55.2 \%, 82.9 \%, 32 \%$, and 92.7\% respectively (Figure 2 ).
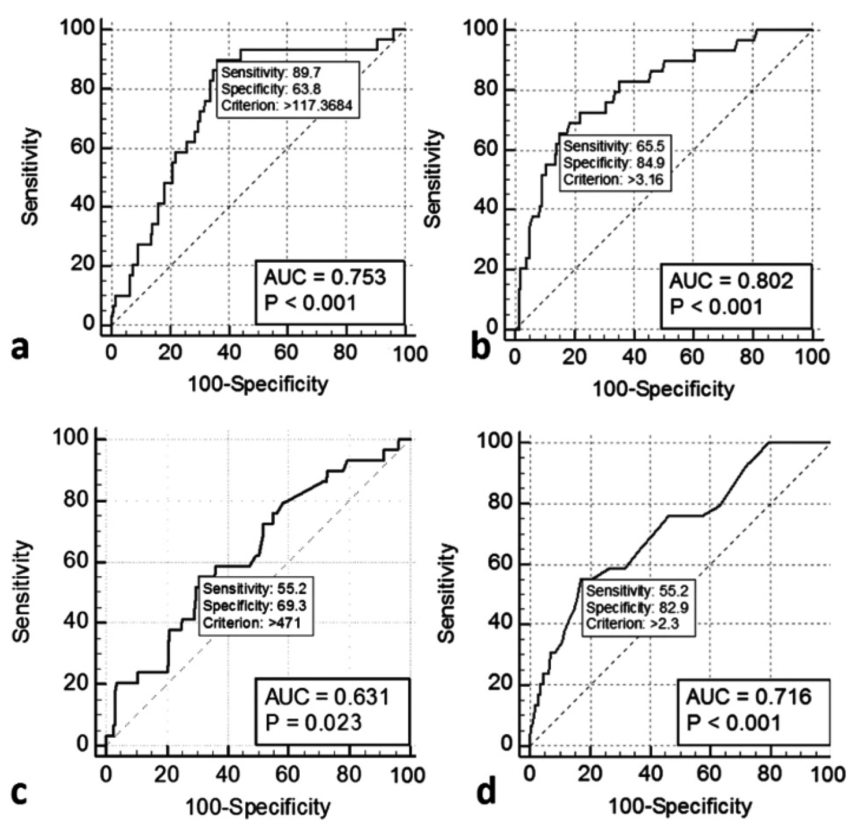

Figure 2: Receiver operating characteristic (ROC) curve of variables (a) Platelet-to-lymphocyte ratio, (b) C-reactive protein, (c) Stone size, (d) Hemoglobin drop.

\section{DISCUSSION}

In this study, the development of postoperative SIRS was investigated retrospectively in patients who underwent PCNL and whose preoperative urine culture was sterile. To the authors' knowledge, the present study is the first in literature to evaluate hematological predictors of SIRS after PCNL in patients who underwent PCNL with a sterile baseline urine culture. This study indicates a significant correlation between inflammation biomarkers and SIRS after PCNL with sterile baseline urine culture. Patients with SIRS after PCNL had higher CRP, PLR and NLR levels than non-SIRS group. Additionally, SIRS group had higher stone size, operative time, blood transfusion, access number, and hospital stay than non-SIRS group. Binary logistic regression test indicated that PLR, CRP, DM, stone size, and hemoglobin drop appeared to be independent useful risk factors of SIRS after PCNL with a sterile baseline urine culture. It was found that high preoperative PRL, high CRP, DM presence, high stone size and high hemoglobin drop had a positive effect on SIRS development.

In this study, which had a broad exclusion criterion, postoperative SIRS was found to develop in a total of 29 (12.7\%) patients. Post- PCNL SIRS incidence varies between $10.49 \%$ and $43 \%$ in literature depending on patient volume. ${ }^{16,17}$ The presently reported SIRS rate is similar to rates in literature. However, considering the strict exclusion criteria, these final SIRS rate was high when compared with the literature.

Despite developing and changing technology, complication rate of PCNL is very high as $11.9 \%$; and SIRS is one of the most common complications after PCNL. ${ }^{3}$ SIRS is closely associated with sepsis and sepsis continues to be the most mortal complication of PCNL. Even if the patient has nega- 
tive preoperative UC and perioperative antibiotic prophylaxis is applied, perioperative fever develops. ${ }^{1}$ In addition, preoperative UC was found to be negative in $36.8-52.4 \%$ of the patients, who developed sepsis after PCNL. ${ }^{8}$ In this case, it is very important to predict SIRS in pre-operative period in patients with sterile UC because detecting and predicting SIRS in post-operative period can prevent urosepsis development and mortality. ${ }^{4}$ This study showed that PRL rate, CRP level, stone size in preoperative period and hemoglobin loss in perioperative period can predict SIRS development. Thus, in the light of these parameters mentioned, the course of patients to sepsis in preoperative period can be predicted and PCNL-induced morbidity and mortality can be decreased with appropriate treatment.

Preoperative and intraoperative risk factors for SIRS development after PCNL have been analysed in a large number of studies. However, there is no consensus on risk factors that predict SIRS after PCNL. Hu et al. reported that female gender, staghorn calculi, urine WBC and positive UC were risk factors of SIRS after PCNL; while age, BMI, DM and surgery time were not. ${ }^{16}$ Tang et al. performed a univariate analysis showing that male gender, blood WBC count, stone burden, hydronephrosis, staghorn calculi, operative time, and stay length were risk factors. ${ }^{11}$ According to Moses et al., age, gender, BMI, ASA, operative time, and preoperative UC are not risk factors. ${ }^{17}$ Univariate analysis showed that $D M$, CRP, PLR, NLR, urine WBC count, stone size, mean ASA score, access number, operative time, mean hemoglobin drop, hospital stay, blood transfusion, and complication rate were related to the development of SIRS but age, gender, $\mathrm{BMI}$, stone location, and SF were not.

In this study, univariate analyses demonstrated that while there was significant difference between the two groups in terms of NLR, and urine WBC values, they were not statistically significant in the multivariate analysis. Tang et al. and $\mathrm{Xu}$ et al. have validated the preoperative NLR as an independent factor for predicting post-PCNL SIRS. ${ }^{11,18}$ However, similar to the results of this study, Cetinkaya et al. reported difference between the two groups in terms of NLR levels. They did not report a difference in multivariate analysis. ${ }^{12}$ Similar to literature, pre-PCNL urine WBC value was found to be associated with the SIRS in this study. However, unlike this study, these results are in agreement with using multivariate analysis in these studies. ${ }^{16,19,20}$

Univariate and multivariate analyses demonstrated the PLR and CRP probability of SIRS after PCNL sterile baseline urine culture. The preoperative PLR ratio and CRP were higher in patients with SIRS than those without SIRS. The cut-off value for PLR was 117.36 which had a sensitivity, specificity, PPV, and NPV of $89.7 \%, 63.8 \%, 26.5 \%$, and $97.7 \%$, respectively. CRP is a sensitive acute phase reactant and inflammation marker, which is inexpensive and noninvasive to measure. Its level in the plasma can rise hundred times more than the normal during bacterial infections. ${ }^{21}$ In our study, the cut-off value for CRP was $3.16 \mathrm{mg} / \mathrm{L}$ which had a sensitivity, specificity, PPV, and NPV of $65.5 \%, 84.9 \%, 38.8 \%$, and $94.4 \%$, respectively. Similar to this study, Ganesan et al. also reported that multivariate analysis indicated that CRP affected the development of SIRS. Because of possible infectious complications, they recommended patients with a CRP $>0.65 \mathrm{mg} / \mathrm{dL} .{ }^{13}$ In this study, the same rate was found as $3.16 \mathrm{mg} / \mathrm{L}$. While Ganesan et al. used turbidimetric immunoassay method to measure CRP level, we used nephelometric immunoassay method in our study. ${ }^{13}$ The authors think that this difference between CRP cut-off values was because they used different measurement method and applied broad exclusion criteria in this study. Given the findings of this study, PLR, and CRP may be an additional biomarker to evaluate as a predictor of SIRS following PCNL especially sterile baseline urine culture.

Another multivariate analysis finding in the present study is the presence of correlation between DM, stone size, hemoglobin drop, and postoperative SIRS. Binary logistic regression analysis found that a hemoglobin drop $>2.3 \mathrm{~g} / \mathrm{dL}$ was an independent risk factor for postoperative SIRS. Similar to this study, Tang et al. and Cetinkaya et al. stated that hemoglobin drop following PCNL was associated with SIRS. However, unlike this study, they reported that hemoglobin drop was not an independent risk factor in multivariate analysis. ${ }^{11,}{ }^{12}$ This study is different from other studies with this aspect. During PCNL operation, bacteria and endotoxins are released from kidney stones. When the size of kidney stone increases, urinary obstruction also increases, surgical procedure becomes more difficult, surgical time increases and hemoglobin loss increases. Due to all these reasons, there is a close relationship between stone size and SIRS after PCNL. ${ }^{6}$ Similar to the results of this study, Chen et al. and Yang et al. reported in their studies that kidney stone size was a risk factor for SIRS after PCNL. ${ }^{8,20}$ In this study, multivariate analysis found that a stone burden of $471 \mathrm{~mm}^{2}$ or greater was a significant predictor of SIRS after PCNL. Since low blood glucose control has a great contribution to infection formation, DM is an independent risk factor for postoperative SIRS development after PCNL. ${ }^{6}$ With this aspect, this study is similar to other studies in literature.

There are several limitations of this study. First of all, its retrospective nature and being a single-centre study may be a disadvantage. Second, more risk factors are not included in this research such as interleukin-6, procalsitonin, pelvis urine cultures, stone cultures, and so on. Third, analgesia, pain, and medications such as beta-blockers are factors that could influence the endpoint of the study by changing heart and/or respiratory rate, this can change the results. For all these reasons, further prospective, multicentre studies are needed.

\section{CONCLUSION}

In the current study, the occurrence of post-PCNL SIRS was $12.7 \%$ in patients with a sterile baseline urine culture. PRL 
$>117.36, \mathrm{CRP}>3.16 \mathrm{mg} / \mathrm{L}$, stone burden $>471 \mathrm{~mm}^{2}$, and hemoglobin drop $>2.3 \mathrm{~g} / \mathrm{L}$ were determined to be independent risk factors for postoperative SIRS after PCNL. Postoperative SIRS and potentially life-threatening sepsis monitorisation should be performed carefully in patients who have these risk factors. In choosing the most appropriate therapy plan for monitorisation of SIRS after PCNL, level of PLR, and CRP should be considered by clinicians before surgery.

\section{ETHICAL APPROVAL:}

All procedures involving human participants were conducted in strict compliance with the ethical principles of the Institutional Research Committee and of the 1964 Declaration of Helsinki and subsequent modifications or equivalent ethical standards. Consent to the use of their clinical data in scientific research was obtained before PCNL from all patients agreeing to the procedure and detailed consent forms were signed by these patients. This study was approved by Health Sciences University, Medical Ethics Committee (Ref. No. GOKA/2020/4/8, Date: 29.01.2020).

\section{PATIENTS' CONSENT:}

Due to the retrospective nature of the study, the data were collected from our hospital archive following receipt of Ethics Committee approval. As required under our hospital regulations, detailed consent forms agreeing to the use of clinical information in scientific research was obtained from all patients prior to PCNL.

\section{CONFLICT OF INTEREST:}

Authors declared no conflict of interest.

\section{AUTHORS' CONTRIBUTION:}

EA: Conception and design of the work, acquisition and analysis of data, drafting the work, final approval.

KO: Acquisition and interpretation of data, literature search, drafting the work, final approval.

MBU: Interpretation and acquisition of data, drafting the work, final approval.

MG: Conception of the work, acquisition of data, drafting the work, final approval.

STC: Design of the work, acquisition of data, drafting the work, final approval.

ES: Conception of the work, analysis of data, literature search, drafting the work, final approval.

\section{REFERENCES}

1. Türk C, Petř́k A, Sarica K, Seits C, Skolarikos A, Straup M, et al. EAU guidelines on interventional treatment for urolithiasis. Eur Urol 2016; 69(3):475-82. doi: 10.1016/ j.eururo.2015.07.041.

2. de la Rosette J, Assimos D, Desai M, Gutierrez J, Lingeman J, Scarpa R, et al.; CROES PCNL study group. The clinical research office of the endourological society percutaneous nephrolithotomy global study: Indications, complications, and outcomes in 5803 patients. J Endourol 2011; 25(1): 11-7. doi: 10.1089/end.2010.0424.
3. Seitz C, Desai M, Häcker A, Hakenberg OW, Liatsikos E, Nagale $U$, et al. Incidence, prevention, and management of complications following percutaneous nephrolitholapaxy. Eur Urol 2012; 61(1):146-58. doi: 10.1016/j.eururo. 2011. 09.016.

4. Singh P, Yadav S, Singh A, Saini AK, Kumar R, Seth A, et al. Systemic inflammatory response syndrome following percutaneous nephrolithotomy: Assessment of risk factors and their impact on patient outcomes. Urol Int 2016; 96(2): 207-11. doi: 10.1159/000441954.

5. O'Keeffe NK, Mortimer AJ, Sambrook PA, Rao PN. Severe sepsis following percutaneous or endoscopic procedures for urinary tract stones. Br J Urol 1993; 72(3):277-83. doi: 10.1111/j.1464-410x.1993.tb00717.x.

6. Chung HS, Jung SI. Perioperative risk factors related to systemic inflammatory response syndrome after percutaneous nephrolithotomy. Urogenit Tract Infect 2019; 14(1): 9-13. doi: 10.14777/uti.2019.14.1.9

7. Kreydin El, Eisner BH. Risk factors for sepsis after percutaneous renal stone surgery. Nat Rev Urol 2013; 10(10): 598-605. doi: 10.1038/nrurol.2013.183.

8. Chen D, Jiang C, Liang X, Zhong F, Huang J, Lin Y, et al. Early and rapid prediction of postoperative infections following percutaneous nephrolithotomy in patients with complex kidney stones. BJU Int 2019; 123(6):1041-7. doi: 10.1111/bju.14484.

9. Fan J, Wan S, Liu L, Zhao Z, Mai Z, Chen D, et al. Predictors for uroseptic shock in patients who undergo minimally invasive percutaneous nephrolithotomy. Urolithiasis 2017; 45(6):573-8. doi: 10.1007/s00240-017-0963-4.

10. Rhodes A, Evans LE, Alhazzani W, Levy MM, Antonelli M, Ferrer $\mathrm{R}$, et al. Surviving Sepsis Campaign: International guidelines for management of sepsis and septic shock: 2016. Intensive Care Med 2017; 43(3):304-377. doi: 10.1007/s00134-017-4683-6.

11. Tang K, Liu H, Jiang K, Ye T, Yan L, Liu P, et al. Predictive value of preoperative inflammatory response biomarkers for metabolic syndrome and post-PCNL systemic inflammatory response syndrome in patients with nephrolithiasis. Oncotarget 2017; 8(49):85612-27. doi: 10.18632/ oncotarget. 20344.

12. Cetinkaya M, Buldu I, Kurt O, Inan R. Platelet-to-lymphocyte ratio: $A$ new factor for predicting systemic inflammatory responsesyndrome after percutaneous nephrolithotomy. Urol J 2017; 14(5):4089-93.

13. Ganesan V, Brown RD, Jimenez JA, De S, Monga M. C-reactive protein and erythrocyte sedimentation rate predict systemic inflammatory response syndrome after percutaneous nephrolithotomy. J Endourol 2017; 31(7):638-44. doi: 10.1089/end.2016.0884.

14. Sen V, Bozkurt IH, Aydogdu O, Yonguc T, Yarimoglu S, Sen $P$, et al. Significance of preoperative neutrophil-lymphocyte count ratio on predicting postoperative sepsis after percutaneous nephrolithotomy. Kaohsiung J Med Sci 2016; 32(10):507-13. doi: 10.1016/j.kjms.2016.08.008.

15. Levy MM, Fink MP, Marshall JC, Abraham E, Angus D, Cook D, et al. 2001 SCCM/ESICM/ACCP/ATS/SIS International Sepsis Definitions Conference. Intensive Care Med 2003; 31(4):1250-6. doi: 10.1097/01.CCM.0000050454.01978.3B. 
16. He Z, Tang F, Lei $H$, Chen $Y$, Zeng G. Risk factors for systemic inflammatory response syndrome after percutaneous nephrolithotomy. Prog Urol 2018; 28(12):582-7. doi: 10.1016/j.purol.2018.06.006.

17. Moses RA, Agarwal D, Raffin EP, Viers BE, Sharma V, Krambeck $A E$, et al. Postpercutaneous nephrolithotomy systemic inflammatory response syndrome is not associated with unplanned readmission. Urology 2017; 100:33-7. doi: 10.1016/j.urology.2016.09.012.

18. Xu H, Hu L, Wei X, Niu J, Gao Y, He J, et al. The predictive value of preoperative high-sensitive c-reactive protein/albumin ratio in systemic inflammatory response syndrome after percutaneous nephrolithotomy. J Endourol 2019;
33(1):1-8. doi: 10.1089/end.2018.0632.

19. Draga RO, Kok ET, Sorel MR, Bosch RJ, Lock TM. Percutaneous nephrolithotomy: factors associated with fever after the first postoperative day and systemic inflammatory response syndrome. J Endourol 2009; 23(6): 921-7. doi:10.1089/end.2009.0041.

20. Yang $T$, Liu $S, H u J$, Wang $L$, Jiang $H$. The evaluation of risk factors for postoperative infectious complications after percutaneous nephrolithotomy. Biomed Res Int 2017; 2017:4832051. doi: 10.1155/2017/4832051.

21. Meisenberg G, Simmons WH. Principles of medical biochemistry. Amsterdam: Elsevier Health Sciences 2011. 